\title{
AN INVARIANT SUBSPACE PROBLEM \\ FOR MULTILINEAR OPERATORS \\ ON FINITE DIMENSIONAL SPACES
}

\author{
JOHN EMENYU
}

\begin{abstract}
We introduce the notion of invariant subspaces for multilinear operators from which the invariant subspace problems for multilinear and polynomial operators arise. We prove that polynomial operators acting in a finite dimensional complex space and even polynomial operators acting in a finite dimensional real space have eigenvalues. These results enable us to prove that polynomial and multilinear operators acting in a finite dimensional complex space, even polynomial and even multilinear operators acting in a finite dimensional real space have nontrivial invariant subspaces. Furthermore, we prove that odd polynomial operators acting in a finite dimensional real space either have eigenvalues or are homotopic to scalar operators; we then use this result to prove that odd polynomial and odd multilinear operators acting in a finite dimensional real space may or may not have invariant subspaces.
\end{abstract}

\section{Introduction}

The aim of this paper is two-fold;

(i) to study the existence of eigenvalues of polynomial operators on finite dimensional spaces.

(ii) to introduce and develop the invariant subspace theory for polynomial and multilinear operators on finite dimensional spaces.

2010 Mathematics Subject Classification. 47A15, 47J10.

Key words and phrases. Invariant subspaces, multilinear operators, polynomial operators, topological degree, admissible operators. 
The notions of invariant subspaces and eigenvalues from which the invariant subspace and eigenvalue problems are derived arise naturally from Science and Engineering fields, see for example, prediction theory [13] and Mathematical Physics [26]. It seems unknown who first stated the invariant subspace problem but can only be backtracked to either after Beurling [6] published his classical paper in Acta Mathematica in 1949 on invariant subspaces of simple shifts in a Hilbert space or after von Neumann's work [25] in 1935 on a proof, by use of spectral theorem for normal operators, that every compact operator on a complex Hilbert space has nontrivial invariant subspaces.

The invariant subspace and eigenvalue theories for bounded linear operators on finite dimensional spaces are fully developed and their usefulness can hardly be overstated; the classical fundamental Burnside theorem [16] and Jordan's structure theorem [7] are some of their outstanding and motivating achievements.

Definition 1.1. Let $\mathcal{M}$ be a closed linear subspace of a space $E$. Then $\mathcal{M}$ is invariant for $T: E \rightarrow E$ if $T(\mathcal{M}) \subseteq \mathcal{M}$, see [5], [7], [22].

The following Problems 1.2 and 1.3 are known as invariant subspace problem and eigenvalue problem for linear operators respectively, see for example [5], $[7],[22]$.

Problem 1.2. Given a bounded linear operator $T \in \mathcal{L}(E)$, does there exist an invariant subspace $\mathcal{M}$ besides the trivial ones $\mathcal{M}=\{0\}$ and $\mathcal{M}=E$ ?

Problem 1.3. Given a bounded linear operator $T \in \mathcal{L}(E)$, does there exist a nonzero vector $v \in E$ such that $T(v)=\lambda v$ for some $\lambda \in \mathbb{k}=\mathbb{C}$ or $\mathbb{R}$ ?

REMARK 1.4. Problems 1.2 and 1.3 have negative answers in some spaces for example the rotation operator $T: \mathbb{R}^{2} \rightarrow \mathbb{R}^{2}$ defined by $T(x, y)=(-y, x)$ does not have eigenvalues as well as nontrivial invariant subspaces. In general, Problem 1.2 has a negative answer in some Banach spaces such as $\ell_{1}$ but is open in others such as Hilbert spaces or more generally reflexive spaces. Moreover, a positive answer to the Problem 1.3 always guarantees a positive answer to Problem 1.2.

Definition 1.5. Let $E$ and $F$ denote Banach spaces. Then a map $T: E \times$ $\ldots \times E \rightarrow F$ is called an $m$-linear map if it is linear in each of the $m$ variables. We denote the space of such continuous maps by $\mathcal{L}\left({ }^{m} E ; F\right)$, see [8].

Notation 1.6. Throughout this paper $\mathbb{k}$ will denote either the field $\mathbb{R}$ of all real numbers or $\mathbb{C}$ the field of all complex numbers. The letters $E$ and $F$ will denote Banach spaces and the use of the term an operator will be restricted to the elements of $\mathcal{L}\left({ }^{m} E ; E\right)$; in this notation the elements of $\mathcal{L}(E)$ are regarded as 1-linear (or linear) operators. 
In Section 2, we will introduce the notion of invariant subspaces for polynomial and multilinear maps. Section 3 contains an overview of the notion of topological degree that plays a major role in the development of invariant subspace theory for polynomial and multilinear operators on arbitrary finite dimensional spaces. Section 4 contains results of our study.

\section{Invariant subspaces for multilinear operators}

In this section, we introduce the notion of invariant subspaces for polynomial and multilinear operators but first we emphasize that any such notion must conform to that of a self map $T: E \rightarrow E$, see Definition 1.1. In this regard, the key idea of our formulation will be derived from the fact that an invariant subspace for a self map $T: E \rightarrow E$ induces another map, namely, the restriction of $T: E \rightarrow E$ to its invariant subspace.

Definition 2.1. A map $p: E \rightarrow E$ is an $m$-homogeneous polynomial if it is the restriction of a map $T \in \mathcal{L}\left({ }^{m} E ; E\right)$ to the diagonal of $E \times \ldots \times E$ (i.e. $p(v)=T(v, \ldots, v)$ for all $v \in E)$. Let $\mathcal{P}\left({ }^{m} E ; E\right)$ denote the Banach space of $m$ homogeneous polynomials, see [8]. A book by Dineen [12] gives an informative and exhaustive literature on polynomial operators and their relationship with multilinear operators.

Definition 2.2. Let $\mathcal{M}$ be a closed linear subspace of $E$ and $p \in \mathcal{P}\left({ }^{m} E ; E\right)$ be the polynomial operator associated to the multilinear operator $T \in \mathcal{L}\left({ }^{m} E ; E\right)$.

(a) Then $\mathcal{M}$ is said to be invariant for $T \in \mathcal{L}\left({ }^{m} E ; E\right)$ if $p(\mathcal{M}) \subseteq \mathcal{M}$ and is strongly invariant for $T \in \mathcal{L}\left({ }^{m} E ; E\right)$ if $T(\mathcal{M}, \ldots, \mathcal{M}) \subseteq \mathcal{M}$.

(b) The subspace of $E$ of the form $\left.\mathcal{M}_{v}=\varlimsup i n p^{n} v: n \in \mathbb{N}\right\}$ for a fixed non-zero $v \in E$ is called an elementary subspace for $T \in \mathcal{L}\left({ }^{m} E ; E\right)$.

The following Problems 2.3 and 2.4 are not only nonlinear analogues of Problems 1.2 and 1.3 respectively but are also their generalizations. We shall in the sequel refer to them as invariant subspace problem and eigenvalue problem for multilinear operators respectively.

Problem 2.3. Let $\mathcal{M}$ be a closed linear subspace of $E$ and $p \in \mathcal{P}\left({ }^{m} E ; E\right)$ be the polynomial associated to the given bounded multilinear operator $T \in$ $\mathcal{L}\left({ }^{m} E ; E\right)$. Then, does there exist an invariant subspace $\mathcal{M}$ for $p \in \mathcal{P}\left({ }^{m} E ; E\right)$ besides the trivial ones $\mathcal{M}=\{0\}$ and $\mathcal{M}=E$ ?

Problem 2.4. Let $p \in \mathcal{P}\left({ }^{m} E ; E\right)$ be the polynomial operator associated to the given bounded multilinear operator $T \in \mathcal{L}\left({ }^{m} E ; E\right)$. Then, does there exist a nonzero vector $v \in E$ such that $p(v)=\lambda v$ for some $\lambda \in \mathbb{k}$ ?

Remark 2.5. The key step in solving Problems 1.2 and 1.3 on finite dimensional spaces is the use of the fundamental theorem of algebra that explores the 
structural (matrix) representation of linear operators, see for example [5, Chapter 1]. Moreover, one can extend the techniques for solving Problems 1.2 and 1.3 on finite dimensional spaces to the Problems 2.3 and 2.4 when the space $E$ is 2-dimensional.

On the other hand, the extension of the techniques for solving Problems 1.2 and 1.3 to the Problems 2.3 and 2.4 when $\operatorname{dim}(E) \geq 3$ faces unprecedented nontrivial difficulties that are caused by the lack of a known matrix representation for $T \in \mathcal{L}\left({ }^{m} E ; E\right)$ and many variants of the notion of the spectrum for nonlinear operators that do not adhere to certain minimum requirements such as the reduction to the usual spectrum for linear operators, the sharing of some topological properties such as compactness and non emptiness, and having relationship with eigenvalues and applications, see [4, Chapters 4-9].

The following Lemma 2.6 and Remark 2.7 will be crucial in the investigation of invariant subspace problem for multilinear operators.

Lemma 2.6. Let $p \in \mathcal{P}\left({ }^{m} E ; E\right)$ be the polynomial operator associated to the multilinear operator $T \in \mathcal{L}\left({ }^{m} E ; E\right)$ where $\operatorname{dim}(E) \geq 2$. If for some $\lambda \in \mathbb{k}$, $p(v)=\lambda v$ then $\operatorname{lin}\{v\}$ is invariant for $p \in \mathcal{P}\left({ }^{m} E ; E\right)$.

Proof. It suffices to show that for any $v \in E$ satisfying the hypothesis of the underlying theorem then $p(u) \in \operatorname{lin}\{v\}$ whenever $u \in \operatorname{lin}\{v\}$. But this is straight forward since if $p \in \mathcal{P}\left({ }^{m} E ; E\right)$ and $u \in \operatorname{lin}\{v\}$, then $p(u)=p(\gamma v)=$ $\gamma^{m} p(v)=\gamma^{m} \lambda v \in \operatorname{lin}\{v\}$.

REMARK 2.7. Let $M$ be a closed linear subspace of $E$. Let $p \in \mathcal{P}\left({ }^{m} E ; E\right)$ be the polynomial associated to the multilinear operator $T \in \mathcal{L}\left({ }^{m} E ; E\right)$. The following are equivalent.

(a) $\mathcal{M}$ is an invariant subspace of $T \in \mathcal{L}\left({ }^{m} E ; E\right)$.

(b) $\mathcal{M}$ is an invariant subspace of $p \in \mathcal{P}\left({ }^{m} E ; E\right)$.

Proof. It is immediate from the Definitions 1.5 and 2.1.

\section{An overview of topological degree of analytic maps}

Consider the invariant subspace problem for $T \in \mathcal{L}\left({ }^{m} \mathbb{k}^{n} ; \mathbb{k}^{n}\right)$. The Lemma 2.6 and Remark 2.7 interrelate the Problems 2.3 and 2.4, that is, the invariant subspace Problem 2.3 turns out to be an eigenvalue Problem 2.4 that are typical existential problems and for $T \in \mathcal{L}\left({ }^{m} \mathrm{k}^{n} ; \mathbb{k}^{n}\right)$ requires knowledge of topological degree theory [2], [19], [24]. The topological degree of $p \in \mathcal{P}\left({ }^{m} \mathbb{k}^{n} ; \mathbb{k}^{n}\right)$ is simply a measure of the number of solutions of the equation $p(v)=u$ where $u \in \mathbb{k}^{n}$.

The notion of the topological degree was introduced by Brouwer [9] in 1912 and later became known as Brouwer degree. He defined the degree of a map $S: \Omega \rightarrow \mathbb{R}^{n}$, denoted by $\operatorname{deg}(S, \Omega, y) \in \mathbb{Z}$, when $\Omega$ is an open bounded subset of $\mathbb{R}^{n}$ and $S$ is a continuous map of $\bar{\Omega}$ into $\mathbb{R}^{n}$ with $y \notin S(\partial \Omega)$. The degree function 
$\operatorname{deg}(S, \Omega, y)$ satisfies certain properties, see [24, Chapter 3], that were extensively studied by different authors such as [3], [15], [17], [18] and was first extended to infinite dimensional spaces by Leray and Schauder [14] in 1934, then to analytic maps such as polynomial maps both in finite and infinite dimensional spaces by different authors, see [10], [11] and [21]. The most important topological degree property in the computation of $\operatorname{deg}(S, \Omega, y)$ that we shall require in this paper is the property of homotopy invariance; it is a global version of continuity. Following a path $H_{t}$ of maps, the number of $y$-points in $\Omega$ can change only if some cross the boundary or else if pairs with indices $\pm k$ (for some $k$ ) coalesce or disjoin.

Consider an analytic map $f: \overline{\mathbf{B}} \rightarrow \mathbb{C}^{n}$ with $y \notin f(\partial \mathbf{B})$ where $\mathbf{B}$ is the open unit ball centered at zero in $\mathbb{C}^{n}$. Let $\left\{\widetilde{z_{1}}, \ldots, \widetilde{z_{n}}\right\}$ be the basis chosen for $\mathbb{C}^{n}$ with $\widetilde{z_{j}}=\widetilde{x_{j}}+i \widetilde{y_{j}}, \widetilde{x_{j}}, \widetilde{z_{j}} \in \mathbb{R}^{n}$ for each $j=1, \ldots, n$. Then, there is a corresponding basis $\left\{x_{1}, y_{1}, \ldots, x_{n}, y_{n}\right\}$ for $\mathbb{R}^{2 n}$; this identification induces an isomorphism between $\mathbf{B}$ and the open unit ball $\Lambda$ centered at the origin in $\mathbb{R}^{2 n}$, between the map $f: \overline{\mathbf{B}} \rightarrow \mathbb{C}^{n}$ and a continuous map $g: \bar{\Lambda} \rightarrow \mathbb{R}^{2 n}$ and between $y \in \mathbb{C}^{n}$ and $\widetilde{y} \in$ $\mathbb{R}^{2 n}$ with $\widetilde{y} \notin g(\partial \Lambda)$, see [21] and references therein. Moreover, for such bases for $\mathbb{C}^{n}$ and $\mathbb{R}^{2 n}, \operatorname{det}\left(J_{g}(x)\right)=\left|\operatorname{det}\left(J_{f}(z)\right)\right|^{2}$, see [11]. Therefore, the Brouwer degree $\operatorname{deg}(g, \Lambda, \widetilde{y})$ is well defined under these conditions and adhere to some topological properties, see for example [24, p. 72]. Subsequently, one can set $\operatorname{deg}(f, \mathbf{B}, y):=$ $\operatorname{deg}(g, \Lambda, \widetilde{y})$ and therefore the topological degree, $\operatorname{deg}(f, \mathbf{B}, y)$, of an analytic map $f$ is well-defined; this degree can be computed by the determinant formula,

$$
\operatorname{deg}(f, \mathbf{B}, y)=\operatorname{deg}(g, \Lambda, \widetilde{y})=\sum_{x \in \mathcal{Z}_{\widetilde{y}}} \operatorname{sign}\left(\operatorname{det} J_{g}(x)\right)
$$

where $\mathcal{Z}_{\widetilde{y}}=g^{-1}(\widetilde{y}) \cap \bar{\Lambda}$ and the sign is always positive. Moreover, the degree $\operatorname{deg}(f, \mathbf{B}, y)$ makes sense if $\operatorname{det} J_{g}(x) \neq 0$ for all $x \in \mathcal{Z}_{\widetilde{y}}$ and is always nonnegative (that is, $\operatorname{deg}(f, \mathbf{B}, y)=0$ if $y \notin f(\overline{\mathbf{B}})$ and $\operatorname{deg}(f, \mathbf{B}, y)>0$ if and only if $y \in f(\overline{\mathbf{B}}))$. The results in [21] are sharp and can be applied to polynomial operators $p \in \mathcal{P}\left({ }^{m} \mathbb{C}^{n} ; \mathbb{C}^{n}\right)$, making topological degree most suitable for investigating Problem 2.4 and therefore Problem 2.3. On the other hand, the results in [10] and [11] give an upper bound on a number of algebraic solutions of the equation $p(z)=0$.

REMARK 3.1. When $n$, the dimension of the space $\mathbb{R}^{n}$, is odd then by the well known hedgehog theorem for Brouwer degree [20, 3.3.26], the Problem 2.4 and subsequently Problem 2.3 has a positive answer; the case $m=1$ is precisely $[1$, Corollary $10.7(1)]$. On the other hand when $n$ is even the hedgehog theorem $[20,3.3 .26]$ fails, see [20, 3.3.27].

The proofs of Problems 1.3 and 2.4 are modeled on the idea of the eigenvalue principle embodied in the following theorem. 
Theorem 3.2. Let $\Omega \subset E$ be bounded and open, and $T: \bar{\Omega} \mapsto E$ be compact vector fields. If $0 \notin S(\partial \Omega), 0 \notin T(\partial \Omega)$ and $\operatorname{deg}(T, \Omega, 0) \neq \operatorname{deg}(S, \Omega, 0)$ then the problem $T z=\lambda S z$ has a solution for $z \in \partial \Omega, \lambda<0$.

\section{Results}

LEMmA 4.1. Every polynomial operator $p \in \mathcal{P}\left({ }^{m} \mathbb{C}^{n} ; \mathbb{C}^{n}\right)$ has eigenvalues.

Proof. If 0 is an eigenvalue of $p \in \mathcal{P}\left({ }^{m} \mathbb{C}^{n} ; \mathbb{C}^{n}\right)$, there is nothing to prove. If not, the degree $\operatorname{deg}(p, \mathbf{B}, 0)$ is well defined, where $\mathbf{B}$ is the open unit ball in $\mathbb{C}^{n}$ centered at zero. We shall tacitly assume below that $m \geqslant 2$ since the case $m=1$ is well known.

Now consider the homotopy $H_{t}: \overline{\mathbf{B}} \times[0,1] \rightarrow \mathbb{C}^{n}$ defined by

$$
H_{t}(z)=(1-t) p(z)+t z \text { for all } t \in[0,1] .
$$

We shall argue that this homotopy is not admissible. If it were so, then by the homotopy invariance of topological degree, $\operatorname{deg}(p, \mathbf{B}, 0)=\operatorname{deg}(I, \mathbf{B}, 0)$. However, by continuity of the topological degree we have $\operatorname{deg}(p, \mathbf{B}, 0)=\operatorname{deg}(p, \mathbf{B}, c)$ if $\|c\|$ is small enough, and by Sard's theorem [23] we may take $c$ to be a regular value so that the latter degree can be computed by the determinant formula. Now, if $p(\omega)=c \neq 0$ and $\gamma_{1}, \ldots, \gamma_{m}$ are the $m$-th roots of unity, then also $p\left(\gamma_{k} \omega\right)=c$ and therefore the sum in the right hand side of the determinant formula is at least $m$. Hence, $\operatorname{deg}(I, \mathbf{B}, 0) \geq m \geq 2$ contradicting the above. Consequently, the homotopy $H_{t}$ cannot be admissible; this means that $H_{t}(z)=0$ for some $t \in(0,1)$ and $z \in \partial \mathbf{B}$. As a result, $p(z)=t z /(t-1)$ so that $p \in \mathcal{P}\left({ }^{m} \mathbb{C}^{n} ; \mathbb{C}^{n}\right)$ must have eigenvalue.

TheOREM 4.2. Every polynomial operator $p \in \mathcal{P}\left({ }^{m} \mathbb{C}^{n} ; \mathbb{C}^{n}\right)$ has nontrivial invariant subspaces.

Proof. By Lemma 4.1, the polynomial operator $p \in \mathcal{P}\left({ }^{m} \mathbb{C}^{n} ; \mathbb{C}^{n}\right)$ has eigenvalues. Then by the Lemma 2.6 , it follows that the polynomial operator $p \in$ $\mathcal{P}\left({ }^{m} \mathbb{C}^{n} ; \mathbb{C}^{n}\right)$ has nontrivial invariant subspaces.

Theorem 4.3. Every multilinear operator $T \in \mathcal{L}\left({ }^{m} \mathbb{C}^{n} ; \mathbb{C}^{n}\right)$ has nontrivial invariant subspaces.

Proof. Let $p \in \mathcal{P}\left({ }^{m} \mathbb{C}^{n} ; \mathbb{C}^{n}\right)$ be the polynomial operator associated to the multilinear operator $T \in \mathcal{L}\left({ }^{m} \mathbb{C}^{n} ; \mathbb{C}^{n}\right)$. Then by Theorem $4.2, p \in \mathcal{P}\left({ }^{m} \mathbb{C}^{n} ; \mathbb{C}^{n}\right)$ has nontrivial invariant subspaces. Therefore, it follows from Remark 2.7 that the multilinear operator $T \in \mathcal{L}\left({ }^{m} \mathbb{C}^{n} ; \mathbb{C}^{n}\right)$ has nontrivial invariant subspaces.

LEMMA 4.4. Every polynomial operator $q \in \mathcal{P}\left({ }^{2 m} \mathbb{R}^{n} ; \mathbb{R}^{n}\right)$ has eigenvalues.

Proof. If 0 is an eigenvalue of $q \in \mathcal{P}\left({ }^{2 m} \mathbb{R}^{n} ; \mathbb{R}^{n}\right)$, then we are done. If not, the degree $\operatorname{deg}(q, \Lambda, 0)$ is well defined, where $\Lambda$ is the open unit ball in $\mathbb{R}^{n}$ centered at zero. 
Now consider the homotopy $h_{t}: \bar{\Lambda} \times[0,1] \rightarrow \mathbb{R}^{n}$ defined by

$$
h_{t}(x)=(1-t) p(x)+t x \quad \text { for all } t \in[0,1] .
$$

We shall again argue that this homotopy cannot be admissible. If it were so, then by the homotopy invariance of topological degree, $\operatorname{deg}(q, \Lambda, 0)=\operatorname{deg}(I, \Lambda, 0)$. However, by continuity of the topological degree, $\operatorname{deg}(q, \Lambda, 0)=\operatorname{deg}(q, \Lambda, c)$ if $\|c\|$ is small enough. Then by Sard's theorem [23] we can consider $c$ to be a regular value so that the latter degree can be computed by the determinant formula. Now, if $q(x) \neq c$ for all $x \in \bar{\Lambda}$ then the sum in the determinant formula is zero and therefore $\operatorname{deg}(q, \Lambda, c)=0$, contradicting the admissibility assumption on the homotopy $h_{t}$. This means that $h_{t}(x)=0$ for some $t \in(0,1)$ and $x \in \partial \Lambda$. Consequently, $q(x)=t x /(t-1)$ so that $q \in \mathcal{P}\left({ }^{2 m} \mathbb{R}^{n} ; \mathbb{R}^{n}\right)$ must have eigenvalues.

However, if $q(\omega)=c$, then also $q(-\omega)=c$. Moreover, by the homogeneity of $q \in \mathcal{P}\left({ }^{2 m} \mathbb{R}^{n} ; \mathbb{R}^{n}\right)$ either $\operatorname{sign}\left(\operatorname{det} J_{q}(\omega)\right)=-\operatorname{sign}\left(\operatorname{det} J_{q}(-\omega)\right.$ or $\operatorname{sign}\left(\operatorname{det} J_{q}(\omega)\right)=$ $\operatorname{sign}\left(\operatorname{det} J_{q}(-\omega)\right)$. Subsequently, by the determinant formula, $\operatorname{deg}(q, \Lambda, 0) \in 2 \mathbb{Z}$ and it is finite. Therefore, again this contradicts the admissibility assumption on the homotopy $h_{t}$. Hence, by the same reasoning as above the polynomial operator $q \in \mathcal{P}\left({ }^{2 m} \mathbb{R}^{n} ; \mathbb{R}^{n}\right)$ must have eigenvalues.

THEOREM 4.5. Every polynomial operator $q \in \mathcal{P}\left({ }^{2 m} \mathbb{R}^{n} ; \mathbb{R}^{n}\right)$ has nontrivial invariant subspaces.

Proof. By Lemma 4.4, the polynomial operator $q \in \mathcal{P}\left({ }^{2 m} \mathbb{R}^{n} ; \mathbb{R}^{n}\right)$ has eigenvalues. Therefore, it follows from Remark 2.7 that the polynomial operator $q \in \mathcal{P}\left({ }^{2 m} \mathbb{R}^{n} ; \mathbb{R}^{n}\right)$ has nontrivial invariant subspaces.

THEOREM 4.6. Every multilinear operator $S \in \mathcal{L}\left({ }^{2 m} \mathbb{R}^{n} ; \mathbb{R}^{n}\right)$ has nontrivial invariant subspaces.

ProOF. Let $q \in \mathcal{P}\left({ }^{2 m} \mathbb{R}^{n} ; \mathbb{R}^{n}\right)$ be the polynomial operator associated to the multilinear operator $S \in \mathcal{L}\left({ }^{2 m} \mathbb{R}^{n} ; \mathbb{R}^{n}\right)$. Then by Theorem 4.5 , the polynomial operator $q \in \mathcal{P}\left({ }^{2 m} \mathbb{R}^{n} ; \mathbb{R}^{n}\right)$ has nontrivial invariant subspaces. Therefore, it follows from Remark 2.7 that the multilinear operator $S \in \mathcal{L}\left({ }^{2 m} \mathbb{R}^{n} ; \mathbb{R}^{n}\right)$ has nontrivial invariant subspaces.

REMARK 4.7. The Lemma 4.4, Theorems 4.5 and 4.6 in general are invalid for polynomial operator $q \in \mathcal{P}\left({ }^{2 m-1} \mathbb{R}^{n} ; \mathbb{R}^{n}\right)$ and multilinear operator $T \in \mathcal{L}\left({ }^{2 m-1} \mathbb{R}^{n} ; \mathbb{R}^{n}\right)$, respectively. A counter example is the following: the polynomial operator $q \in \mathcal{P}\left({ }^{3} \mathbb{R}^{2} ; \mathbb{R}^{2}\right)$ defined by $q\left(x_{1}, x_{2}\right)=\left(-x_{2}^{3}, x_{1}^{3}\right)$ does not have real eigenvalues as well as nontrivial invariant subspaces. In fact, this is a general phenomenon exhibited by odd $m$-linear operators that are homotopic to scalar operators. A scalar operator is one that is a complex multiple of the identity.

Below, we shall only consider Problem 2.3 for $q \in \mathcal{P}\left({ }^{2 m-1} \mathbb{R}^{2 n} ; \mathbb{R}^{2 n}\right)$ since the existence of nontrivial invariant subspaces for $q \in \mathcal{P}\left({ }^{2 m-1} \mathbb{R}^{2 n-1} ; \mathbb{R}^{2 n-1}\right)$ is 
guaranteed by the well known hedgehog theorem for Brouwer degree [20, 3.3.26], Lemma 2.6 and Remark 2.7.

LEMma 4.8. A polynomial operator $q \in \mathcal{P}\left({ }^{2 m-1} \mathbb{R}^{2 n} ; \mathbb{R}^{2 n}\right)$ either has eigenvalues or is homotopic to a scalar operator.

Proof. If 0 is an eigenvalue of $q \in \mathcal{P}\left({ }^{2 m-1} \mathbb{R}^{2 n} ; \mathbb{R}^{2 n}\right)$ then we are done. If not, the degree $\operatorname{deg}(q, \Lambda, 0)$ is well defined and $\operatorname{deg}( \pm I, \Lambda, 0)=( \pm 1)^{2 n}=1$.

Consider the homotopy $h_{t}: \bar{\Lambda} \times[0,1] \rightarrow \mathbb{R}^{n}$ defined by

$$
h_{t}(x)=(1-t) q(x)+t x \quad \text { for all } t \in[0,1] .
$$

Now by continuity of the topological degree, we have $\operatorname{deg}(q, \Lambda, 0)=\operatorname{deg}(q, \Lambda, c)$ if $\|c\|$ is small enough. By Sard's theorem [23] take $c$ to be a regular value so that the latter degree can be computed by the determinant formula. Now the ball $\bar{\Lambda}$ is always symmetric about its center at zero and therefore by BorsukUlam theorem [24, p. 78], there is always $x_{0} \in \bar{\Lambda}$ such that $q\left(x_{0}\right)=c$ since if it was not so, the determinant formula would give $\operatorname{deg}(q, \Lambda, 0)=0 \in\{2 m-1$ : $m \in \mathbb{N}\}$ which is absurd. However, $q\left(-x_{0}\right)=-q\left(x_{0}\right)=-c \neq c$. Therefore, the determinant formula yields either $\operatorname{deg}(q, \Lambda, c)=1$ or $\operatorname{deg}(q, \Lambda, c) \in\{2 m+1$ : $m \in \mathbb{N}\}$. Subsequently, in the case $\operatorname{deg}(q, \Lambda, c)=1$, the polynomial operator $q \in$ $\mathcal{P}\left((2 m-1) \mathbb{R}^{2 n} ; \mathbb{R}^{2 n}\right)$ is homotopic to a scalar operator. Otherwise, $h_{t}$ cannot be admissible and so the operator $q \in \mathcal{P}\left({ }^{(2 m-1)} \mathbb{R}^{2 n} ; \mathbb{R}^{2 n}\right)$ must have eigenvalues.

THEOREM 4.9. Every polynomial operator $q \in \mathcal{P}\left({ }^{2 m-1} \mathbb{R}^{2 n} ; \mathbb{R}^{2 n}\right)$ that is not homotopic to a scalar operator has nontrivial invariant subspaces.

Proof. By the Lemma 4.8, the polynomial operator $q \in \mathcal{P}\left({ }^{2 m-1} \mathbb{R}^{2 n} ; \mathbb{R}^{2 n}\right)$ has eigenvalues. Therefore, it follows from Lemma 2.6 that the polynomial operator $q \in \mathcal{P}\left({ }^{2 m-1} \mathbb{R}^{2 n} ; \mathbb{R}^{2 n}\right)$ has nontrivial invariant subspaces.

THeOREM 4.10. Let $S \in \mathcal{L}\left({ }^{2 m-1} \mathbb{R}^{n} ; \mathbb{R}^{n}\right)$ and $q \in \mathcal{P}\left({ }^{2 m-1} \mathbb{R}^{n} ; \mathbb{R}^{n}\right)$ be its associated polynomial operator that is not homotopic to a scalar operator. Then the multilinear operator $S \in \mathcal{L}\left({ }^{2 m-1} \mathbb{R}^{n} ; \mathbb{R}^{n}\right)$ has nontrivial invariant subspaces.

Proof. By Theorem 4.9 and the well known hedgehog theorem for Brouwer degree [20,3.3.26], $q \in \mathcal{P}\left({ }^{2 m-1} \mathbb{R}^{n} ; \mathbb{R}^{n}\right)$ has nontrivial invariant subspaces. Therefore, it follows from Remark 2.7 that the multilinear operator $S \in \mathcal{L}\left({ }^{2 m-1} \mathbb{R}^{n} ; \mathbb{R}^{n}\right)$ has nontrivial invariant subspaces.

REMARK 4.11. The arguments of the proofs of our results are valid in any normed space $E$ of dimension $n$ since for a chosen basis for $E$, one can identify $E$ with $\mathbb{k}^{n}$ in a natural fashion. Moreover, the maps $f: \mathbb{k}^{n} \rightarrow \mathbb{k}^{n}$ and $F: E \rightarrow E$ are similar, that is, there exists a homeomorphism $h: E \rightarrow \mathbb{k}^{n}$ such that $F=h f h^{-1}$, see for example [19] and references therein. The conclusion then follows from the fact that similarity preserves invariant subspaces. We, therefore, state the following theorems without giving their proofs. 
TheORem 4.12. Let $E$ be a finite dimensional complex space. Then every multilinear $T \in \mathcal{L}\left({ }^{m} E ; E\right)$ has nontrivial invariant subspace in $E$.

Theorem 4.13. Let $E$ be a finite dimensional real space. Then every multilinear operator $T \in \mathcal{L}\left({ }^{2 m} E ; E\right)$ has nontrivial invariant subspace in $E$.

REMARK 4.14. Theorems 4.5, 4.6, 4.9, 4.10 characterize multilinear operators and their associated polynomial operators into two major subclasses;

(a) even multilinear operators and their associated polynomial operators that have nontrivial invariant subspaces.

(b) odd multilinear operators and their associated polynomial operators that may or may not have nontrivial invariant subspaces; this includes linear operators.

Moreover, these results show that the existence of invariant subspaces for a given operator depends both on the linearity structure of the operator (see Theorems 4.5 and 4.6) and dimensional structure of the space (see Theorems 4.9 and 4.10). On the other hand, [1, Corollary 10.7], shows that the dimensional structures of the space play a more subtle role in the existence of invariant subspaces for operators acting on them. This does not only point to the fact that the development of any robust method for solving an invariant subspace problem affirmatively should be based on both the structure of the space and operator but also the difficulties that one faces in solving the problem itself.

Acknowledgement. I thank the Analysis research team at Freie Universität, Berlin for their various contributions to this research. I also thank DAAD for the financial support towards my stay in Germany. Finally, I thank the referee for pointing out the use of hedgehog theorem in determining eigenvalues of an operator on an odd dimensional space.

\section{REFERENCES}

[1] Y.A. Abramovich and C.D. Aliprantis, An invitation to operator theory. Graduate studies in Mathematics, Amer. Math. Soc. 50.

[2] R.P. Agarval, M. Meehan and D. O'Rregan, Fixed point theory and applications, Cambridge Tracts in Math. 141 (2001), Cambridge University Press, Cambridge.

[3] H. Amann And S.A. Weiss, On the uniqueness of the topological degree, Math. Z. 130 (1973), 39-54.

[4] J. Appell, E. De Pascale and A. Vignoli, Nonlinear Spectral Theory, de Gruyter Series in Nonlinear Analysis and Applications, vol. 10, de Gruyter-Verlag, Berlin, 2004.

[5] B. BEAUZAmy, Introduction to Operator Theory and Invariant Subspaces, North-Holland Mathematical Library, vol. 42, 1998.

[6] A. Beurling, On two problems concerning linear transformations in Hilbert spaces, Acta Math. 81 (1949), 239-255. 
[7] H. Bercovivi, Notes on invariant subspaces, Bull. Amer. Math. Soc. 23 (1990), 1-36.

[8] F. Bombal and M. Fernandez, Polynomial properties and symmetric tensor product of Banach spaces, Arch. Math. 74 (2000), 40-49.

[9] L.E.J. Brouver, Über Abbildung von Mannigfaltigkeiten, Math. Ann. 71 (1912), 97115.

[10] F.E. Browder, Nonlinear operators in Banach spaces, Math. Ann. 162 (1966), 280283.

[11] J. Cronin, Analytic functional mappings, Ann. Math. 58 (1953), 175-181.

[12] S. Dineen, Complex analysis on infinite-dimensional spaces, Monograph in Mathematics, Springer-Verlag London, 1990.

[13] A.N. Kolmogorov, Interpolation and extrapolation, Bull. Acad. Sci. USSR Sér. Math. (1941), 3-14.

[14] J. Leray and J. Sschauder, Topologie et équations fonctionnelles, Ann. Sci. Ecole. Norm. Sup. 51 (1934), 45-78.

[15] N.G. Lloyd, A survey of degree theory: Basis and development, IEEE Trans. Circuits and Systems CAS 30 (1983), 607-616.

[16] V.I. Lomonosov, An extension of Burnside's theorem to infinite dimensional spaces, Israel J. Math. 75 (1991), 329-333.

[17] M. Nagumo, A note on the theory of degree of mapping in Euclidean spaces, Osaka Math. J. 4 (1952), 1-9.

[18] A theory of degree of mapping based on infinitesimal analysis, Amer. J. Math. 73 (1951), 485-496.

[19] J. OINAS, The degree theory and the index of a critical point for mappings of the type (S+), PhD, Acta Univ. of Oulu A. 488 (2007), http://herkules.oulu.fiisbn9789514284878/.

[20] N.S. Papageorgius and S.T. Kyrtsi-Yiallourou, Handbook of Applied Analysis, Advances in Mechanics and Mathematics, vol. 19, Springer, Dordrecht, Heidelberg, London, New York, 2009.

[21] P.H. Rabinowitz, A note on topological degree theory for holomorphic maps, Israel J. Math. 16 (1973), 46-52.

[22] H. Radjavi and P. Rosenthal, Invariant subspaces, 2nd ed., Springer-Verlag, Berlin, New York, 1973.

[23] A. SARD, The measure of the critical values of differentiable maps, Bull. Amer. Soc. 48 (1942), 883-890.

[24] J.T. Schwartz, Nonlinear Functional Analysis, Lecture Notes, New York University, Courant Institute of Mathematical Sciences, 1965.

[25] J. von Neumann, Charakterisierung des Spectrum Cines Integraloperators, Herman, Paris, 1935.

[26] N. Wiener, Extrapolation and Smoothing of Stationary Times Series, New York, 1949.

Manuscript received December 21, 2011

JOHN EMENYU

Mbarara University of Science and Technology

Department of Mathematics

P.O. Box 1410 Mbarara, UGANDA

E-mail address: jemenyu@must.ac.ug, john.emenyu@fulbrightmail.org

TMNA : Volume $44-2014-\mathrm{N}^{\mathrm{o}} 1$ 